\title{
Práticas Educativas Para a Prevenção do HIV/AIDS: Aspectos Conceituais
}

\section{Conceptual Aspects of Educational Practices for HIV/AIDS Prevention}

\author{
João Claudio L. Fernandes ${ }^{1}$
}

\begin{abstract}
FERNANDES, F. C. L. Conceptual Aspects of Educational Practices for HIV/AIDS Prevention. Cad. Saúde Públ., Rio de faneiro,10 (2): 171-180,Apr/fun, 1994.

The importance and complexity of educational work related to HIV prevention demand a continuing discussion of its contents. Regarding the ideologic features, it must be assumed that, with the AIDS epidemic, medical science reaches a dimension of the life relatively preserved from its previous hegemonic position: sexual behavior. In this field, a coercive attitude on the part of health professionals may lead to negative reactions. To avoid this, individual and community participation and selfresponsibility are central tools for the improvement of the efficacy of education practices.

The author proposes three components that educational planners should consider: information and its effects on knowledge, attitudes, and perception related to HIV infection; response facilitation; and support for individual and collective reflection.
\end{abstract}

Keys words: Health Education; AIDS; HIV; Prevention

\section{INTRODUÇÃO}

A epidemia do HIV representa uma das mais sérias ameaças à saúde pública dos nossos tempos. Na falta de métodos preventivos e curativos eficazes no campo da biologia, as possibilidades de combate à sua propagação continuam sendo amplamente sustentadas no trabalho educativo, visando estimular os riscos de infecção. Esta realidade demanda o constante aprofundamento do debate e da reflexão em torno das práticas educativas, de modo a aumentar sua eficácia e apontar caminhos que respondam aos diversos desafios a elas relacionados.

De fato, a questão da mudança de hábitos, particularmente nos campos diretamente implicados na transmissão do HIV, está bem longe de ser simples e transcende, sem dúvida, o aspecto estritamente informativo, como pode ser observado nos resultados obtidos em diver-

${ }^{1}$ Posto de Saúde da Associação dos Moradores e Amigos do Bairro Barcellos. Travessa Flores, 4, Favela da Rocinha, Rio de Faneiro, RF, 22451-410, Brasil. sos estudos sobre conhecimentos e atitudes relacionados à infecção. Em uma pesquisa realizada na favela da Rocinha, Rio de Janeiro, Brasil, por exemplo, observou-se entre os entrevistados um nível bastante razoável de conhecimentos sobre a doença, com mais de $95 \%$ de respostas corretas em relação às formas de transmissão sexual (Fernandes et al., 1992). Cerca de $45 \%$ dos entrevistados, entretanto, acreditavam que a picada de mosquito poderia transmitir o vírus, e apenas $8 \%$ deles referiram usar regularmente o preservativo. A mesma opinião sobre o mosquito enquanto vetor da infecção foi compartilhada por $57 \%$ dos entrevistados em um estudo feito numa população de 100 mil norte-americanos (Médecine et Hygiène, 1991). Em um outro inquérito realizado entre usuários do metrô de São Paulo, registrou-se uma referência a mudanças, enquanto somente $18 \%$ deles referiram o uso de preservativo (Munhoz et al., 1992). Parker (1991), estudando uma população de homens homo e bissexuais, relata um amplo conhecimento da importância do preservativo como meio de prevenção da infecção, aliado, no entanto, ao uso inconsciente deste. 
Estes resultados apontam para a existência de fatores, para além da informação específica sobre a AIDS ou subjacentes a ela, que interferem na adoção de comportamentos preventivos eficazes e que devem ser cuidadosamente investigados e considerados no planejamento de trabalhos educativos. Disto podemos depreender que a eficiência das atividades de educação dependerá fortemente do nível de abrangência e contextualização com que estas forem planejadas e desenvolvidas.

Este texto pretende colaborar no levantamento de pontos potencialmente correlacionados ao desempenho destas atividades.

\section{ASPECTOS IDEOLÓGICOS DA EDUCAÇÃO PARA A AIDS}

Um primeiro ponto a ser considerado refere-se aos conteúdos ideológicos ligados às práticas de educação, ainda pouco discutidos no contexto da AIDS. Podemos dizer que, de um modo geral, existe um conteúdo medicalizador e autoritário embutido em maior ou menor grau nas práticas de medicina preventiva. Boltanski (19889: 45) refere que

"a atitude autoritária do médico frente aos membros das classes populares... nunca aparece tão claramente quanto no exercício da medicina preventiva, 'medicina da saúde', que exige, no entanto, para se completar totalmente, uma estreita colaboração entre o médico, encarregado de difundir as regras de higiene, e aqueles que devem pô-las em prática”.

Se, por um lado, esta atitude alcança, em alguns campos, uma certa eficácia na persuasão e adesão das pessoas aos preceitos médicos, pode, por outro, despertar reações contra-hegemônicas que acabam por dificultar a elaboração de comportamentos pessoais saudáveis. Rifkin a\& Walt (1986: 564), em um estudo sobre duas diferentes formas de práticas de saúde, apontam para a ineficácia de projetos autoritários, que não consideram "o longo e lento processo que conduz à melhoria sustentada da vida das pessoas".
No campo específico da infecção pelo HIV é forçoso reconhecer que, com a epidemia, a medicina alcança um campo de intervenção social que lhe era até então relativamente restrito: a vida sexual das pessoas. A partir de então, ela passa a normatizar quantidades, parcerias e formas de relação sexual permitidas, definindo, muitas vezes de modo claramente coercitivo, novos padrões de comportamento sexual. Ou, atualizando a afirmação de Clavreul (1983: 47), "regulamentando a vida e o gozo, e negando qualquer outra razão de viver que não seja a razão médica que faz viver, eventualmente à força”. Esta intenção hegemônica se manifesta através de posturas muitas vezes sutis e de difícil visualização, mas com repercussões profundas tanto no campo da ética quanto, como comentamos anteriormente, na própria eficácia técnica das atividades propostas.

É preciso ter claro que, se por um lado interessa aos serviços de saúde e ao Estado o controle da infecção, é fundamental reconhecer clara e explicitamente a responsabilidade e livre-arbítrio dos cidadãos em definir a conduta que lhes convém em relação aos riscos de contaminação. Não queremos, com esta afirmação, desconsiderar nem os aspectos mais gerais que interferem no comportamento das pessoas, nem as questões éticas implicadas na decisão comportamental. Não se trata, portanto, de cristalizar uma abordagem individualista. Pretendemos aqui, sobretudo, reforçar um enfoque que julgamos importante, e freqüentemente negado, no desempenho das práticas educativas: a importância do reconhecimento da auto-responsabilidade, mesmo admitindo que este tempo guarda uma série de indefinições e ambigüidades. Entendemos que cabe aos organismos responsáveis convidar as pessoas para a discussão, oferecendo informações para seu julgamento e apreciação, tratando-as, para usar a imagem de Paulo Freire (1975), não como "vasilhas a serem enchidas", mas como sujeitos, portadores de uma cultura e historicidade e proprietários de seu destino. É, portanto, dentro deste contexto ideológico que as questões mais gerais, da ordem da cultura e das relações sociais existentes, poderão ser identificadas e abordadas. Parece-nos que, se 
estes princípios são válidos para quaisquer atividades educativas, em relação à AIDS se tornam essenciais, na medida em que as decisões de mudança possuem um caráter especialmente íntimo, particular e sensível, e, portanto, dependentes de processos autênticos de internalização e elaboração.

Em outras palavras, se a necessidade de mudanças de atitudes na vida sexual é percebida como uma imposição de fora, obrigando a restrições num campo até então considerado "livre", onde a regra, como refere Parker a(1987), é a do "vale tudo", e que vinha alcançando conquistas cada vez maiores na sociedade, é razoável esperar que ocorram reações, também dirigidas ao impositor externo, e não às próprias resistências pessoais. Desta forma, as práticas educativas que objetivam a modificação de comportamentos não devem ser justificadas apenas "na medida em que não reduzem a dignidade e auto-responsabilidade", como sugerem McAlister et al. (1991: 3), mas exatamente de modo a considerar o processo de responsabilização e participação pessoal e coletiva como ponto essencial da sua estratégia.

Para ilustrar esta questão, julgamos interessante levantar alguns pontos referentes à política de controle da AIDS em Cuba. Este país diferenciou-se dos demais ao adotar precocemente uma ampla política de controle baseada na testagem compulsória de grupos populacionais cada vez mais abrangentes, na prática da sanatorização de soropositivos e doentes, além do trabalho educativo voltado para a população (DNE/Minsap, 1992). Não nos cabe aqui avaliar os eventuais impactos desta política no campo epidemiológico ou ético. Queremos, sobretudo, destacar alguns possíveis efeitos dela sobre as práticas educativas. Santana et al. (1992), que realizaram um estudo de natureza qualitativa do Programa Nacional de Prevenção e Controle da AIDS (PNPCA) cubano entre 1986 e 1990, observaram, entre outros aspectos, que muitas pessoas pareciam sentir que estavam protegidas pelas medidas tomadas para isolar os soropositivos, não precisando, deste modo, modificar seus hábitos sexuais. A partir desta observação a autora questiona até que ponto o regime sanatorial foi importante como forma de prevenção de novas infecções, ou contraproducente por reforçar um comportamento sexual pouco seguro na população. Embora este dado não possa ser considerado representativo da opinião da maioria da população cubana - e por isto não se trata aqui de avaliar aquele programa em si-, ele traz à tona um possível subproduto de uma ação educativa desenvolvida em um contexto autoritário que poderá, eventualmente, reduzir chances do esperado impacto positivo daquele programa.

A partir destas colocações, podemos deduzir que este aspecto do trabalho educativo tem também uma relação direta com os custos e a autosustentação das atividades, no contexto social. Como referem Green \& Krenter (1991: 269),

"pedir a comunidades e às organizações que implementem programas planejados em outros lugares e avaliados nos termos definidos por outrem pode ganhar alguns adeptos, mas freqüentemente com compromissos apenas enquanto durar o dinheiro".

Uma última questão a ser considerada neste ponto é que a importância do reconhecimento do direito e responsabilidade de cada cidadão em definir seu comportamento perante a epidemia conduz à necessidade, como contrapartida, de um posicionamento também claro por parte dos planejadores de saúde e do Estado sobre suas próprias e específicas responsabilidades. Para garantir a credibilidade das práticas educativas relacionadas à AIDS, estas devem estar inseridas em um conjunto de atividades inter-reforçadoras, que caracterizem políticas de controle. Isto significa, por exemplo, desenvolver um sistema que garanta a produção de informações confiáveis em relação à situação da epidemia no país e ao seu perfil epidemiológico, de modo a prover substratos consistentes nos quais a população possa basear suas avaliações; garantir o acesso aos meios de prevenção, bem como a serviços de testagem e tratamento de boa qualidade etc. Estas são algumas responsabilidades que o Estado e os profissionais de saúde precisam assumir, de modo a se mostrarem realmente preocupados 
com a ameaça que a epidemia representa e criar um ambiente favorecedor da conscientização popular e responsabilização pessoal e coletiva.

\section{FACILITAÇÃO}

Dentro do campo educativo, estas questões constituem o que Bandura chama de facilitação da resposta (McaAlister et al., 1991). Um aspecto particularmente sensível desta facilitação, que queremos destacar, refere-se aos métodos de prevenção, amplamente baseados na utilização de preservativos cujo preço, tratando-se de um país pobre, é um viés intransponível para ações de prevenção exclusivamente informativas. Uma caixa com três preservativos custa atualmente em torno de CR $\$ 12.000,00$ (US\$ 1.7), o que equivale ao preço de 4,2 litros de leite (9/92, Rio de Janeiro). É possível supor que existe um certo número de pessoas que usariam de bom grado o preservativo, sem a necessidade de grandes discussões, ou em função do que já sabem sobre a AIDS, ou por intenções contraceptivas, e que simplesmente não podem adquiri-lo. Ouvimos diversas vezes o relato de jovens que diziam ter de escolher, ao ir a uma festa, entre tomar um chope ou comprar camisinhas. A decisão é compreensível.

As atividades educativas devem, portanto, considerar seriamente estes aspectos e elaborar estratégias que visem garantir o acesso regular de seu público aos meios facilitadores. Uma alternativa que utilizamos, aparentemente com sucesso, é a distribuição periódica controlada de preservativos, através de pessoas do próprio grupo ou comunidade, feita a partir do cadastramento dos interessados (PS.AMABB, 1991). Outra alternativa seria a distribuição baseada em instituições acessíveis. Na medida em que a mudança de hábitos é um processo, a regularidade e a comunidade destes mecanismos podem ser pontos decisivos para se alcançar os objetivos desejados. Neste sentido, deve-se considerar as distribuições eventuais de preservativos em palestras ou em determinados eventos como atividades limitadas do ponto de vista estratégico, embora, como veremos adiante, úteis eventualmente em termos táticos.

\section{AQUESTÃO DOS GRUPOSDERISCO}

Uma das conseqüências da adoção de um modelo teórico autoritário nas práticas educativas é sua transposição mecânica a contextos diferenciados, já que não considera, na prática, a participação das pessoas na sua elaboração e desenvolvimento. Em relação a este aspecto, uma questão a ser avaliada são as estratégias dirigidas especificamente a determinados subgrupos populacionais, baseadas na premissa de que o controle da infecção dependeria de uma atuação seletiva nestes grupos. Estas estratégias têm sido amplamente preconizadas nos Estados Unidos da América e adotadas em diversos outros países. Para discutirmos esta questão, o primeiro ponto a ser confrontado é o fato epidemiológico em si. Existe no Brasil, como em quase todo o mundo, uma nítida tendência da epidemia de atingir cada vez mais intensamente a população em geral, representada particularmente, neste momento, pelas mulheres (MS, 1992). Em segundo lugar, a abrangência e o significado das denominações usadas para caracterizar estes subgrupos "de alto risco" podem variar intensamente entre diferentes culturas. Parker (1978), por exemplo, faz um interessante estudo sobre os conteúdos atribuídos ao termo "homossexual" no Brasil, confrontando-o com os conceitos de "atividade" e a "passividade". Segundo o autor, o homossexual, nesta cultura, entendido como o não-masculino (bicha), seria representado por pessoas engajadas em práticas homossexuais receptivas. As práticas penetrativas são consideradas genuinamente masculinas, independente do sexo do parceiro. É fácil imaginar as conseqüências práticas desta "falha de percepção" por parte dos veiculadores de informações seletivas. Por último, concordamos com as preocupações levantadas por Rifkin a\& Walt (1986), de que a atuação seletiva em determinados grupos não implica necessariamente na inexistência de repercussões para os demais, que podem implicitamente se considerar com menos importância ou menor risco de infecção e sedimentar hábitos inadequados à prevenção da doença. Desta forma, o objetivo do trabalho educativo deve ser a comunidade e todos os seus membros (Green \& Krenter, 1991) e é a partir deste 
contexto que podem ser organizadas atividades dirigidas especificamente a determinados grupos.

\section{CAMPOS DE PRÁTICA EDUCATIVA}

A partir destas observações, podemos considerar algumas questões mais especificamente ligadas às diferentes formas da prática educativa. Apesar da importância que atribuímos anteriormente à regularidade e continuidade do trabalho, as atividades educativas instantâneas, como debates, campanhas, distribuições eventuais de preservativos, podem ser também de utilidade, desde que reconhecidos seus limites e, portanto, assumidas suas possibilidades de impacto. Na verdade, uma questão a ser levada em conta é a da diversidade de métodos e práticas que podem atuar sinergicamente para a aquisição de hábitos preventivos. Existe um vasto leque de possibilidades de atuação, cada uma com suas limitações e potencialidades específicas e cujo conjunto pode influir pesadamente no controle da epidemia. A integração de atividades massivas, grupais e individuais, continuadas e instantâneas, dentro de um contexto participativo, pode ser desenvolvida com um custo relativamente reduzido e favorecer, por vários lados, a conscientização e o movimento de adaptação das pessoas à nova realidade. A importância desta diversificação relaciona-se à própria natureza e complexidade do processo educativo e visa, em última análise, discutir e buscar a mudança nas normas sociais que encorajam o engajamento de pessoas em comportamentos de risco (Kelly \& Lawrence, 1990) e, por outro lado, favorecer a reflexão e decisão individuais.

Exemplificando, podem ser planejadas atividades dirigidas a uma comunidade ou município como um todo, como comemoração do dia mundial de luta contra a AIDS, shows etc, aliadas à utilização apropriada da mídia e às chamadas atividades de marketing social, associadas, por sua vez, a encontros grupais etc. A participação da comunidade no planejamento destas ações, não custa insistir, é crucial em termos de factibilidade, efetividade e eficiência.

Em relação às práticas grupais, existe uma série de dinâmicas que podem ser úteis ao trabalho educativo, como os sociodramas ligados a situações de risco (pode-se abordar, por exemplo, a recusa do parceiro em usar o preservativo, o uso de álcool ou de drogas antes da atividade sexual etc) (Kelly et al., 1989; Kelly \& Lawrence, 1990). A metodologia de Paulo Freire (Freire, 1975; 1978) também pode ser adaptada, utilizando-se histórias, palavras ou situações relacionadas ao problema para gerar a reflexão no grupo.

Estas práticas, por sua vez, produzem importantes multiplicadores para a conversa interpessoal. Em relação a este aspecto existe uma ampla aceitação de que a ação de pessoas do próprio grupo ou da comunidade otimiza os efeitos e facilita a continuidade do processo educativo (Peterson \& Szterenfeld, 1992; McAlister, 1991; O’Reilly, 1991; Green \& Krenter, 1991). Existem, entretanto, algumas variações na estratégia utilizada: alguns propõem uma atuação mais profissionalizada com agentes comunitários, geralmente remunerados, enquanto outros sugerem a ação de moradores mais como modelos ou referências para os demais. Na nossa opinião, embora ambas as formas de atuação devam ser pensadas em função da realidade específica de cada trabalho, deve-se levar em conta que uma atuação menos profissionalizada pode ter um melhor efeito no sentido da normalização da informação sobre AIDS, apontando mais claramente para a perspectiva de que cada parente, cada colega, cada pessoa pode e deve ser uma fonte de educação. É possível também que a atuação de educadores profissionais, mesmo sendo da própria comunidade, provoque uma impressão de cooptação e diferença em relação aos demais, passando estes a ser identificados (e também a se identificar!) ideologicamente mais com a instituição ou com o Estado que os contrata que com seus companheiros.

\section{OS COMPONENTES DAS PRÁTICAS EDUCATIVAS}

Em relação aos conteúdos do trabalho educativo, consideramos a existência de três aspectos fundamentais, já mencionados em ou- 
tras partes deste texto, a serem avaliados no seu planejamento: a informação a ser oferecida, a facilitação da resposta, e o favorecimento da abertura de espaços para a reflexão e discussão (Figura 1). Estes aspectos diferem relativamente dos conceitos desenvolvidos por McAlister et al. (1991), baseados na teoria do aprendizado social de Bandura, na medida em que não separamos a informação dos fatores ligados à persuasão e motivação. Como já mencionamos anteriormente, é fundamental que as pessoas sejam confrontadas com sua capacidade de reflexão e responsabilidade de decisão; portanto, devemos ter cautela em relação a estratégias voltadas especificamente para aa persuasão e motivação, como, por exemplo, o uso de prêmios ou recompensas. Não queremos com isto propor uma pretensa neutralidade do educador, já que a informação por ele veiculada carregará sempre um determinado conteúdo capaz de influenciar subjetivamente o comportamento, como veremos adiante. Portanto, será sempre apresentada com conteúdos mais ou menos persuasivos, ou mais ou menos motivadores. Como diria Torquato Neto, " $a$ pureza é um mito".

FIGURA 1. Componentes do Trabalho Educativo

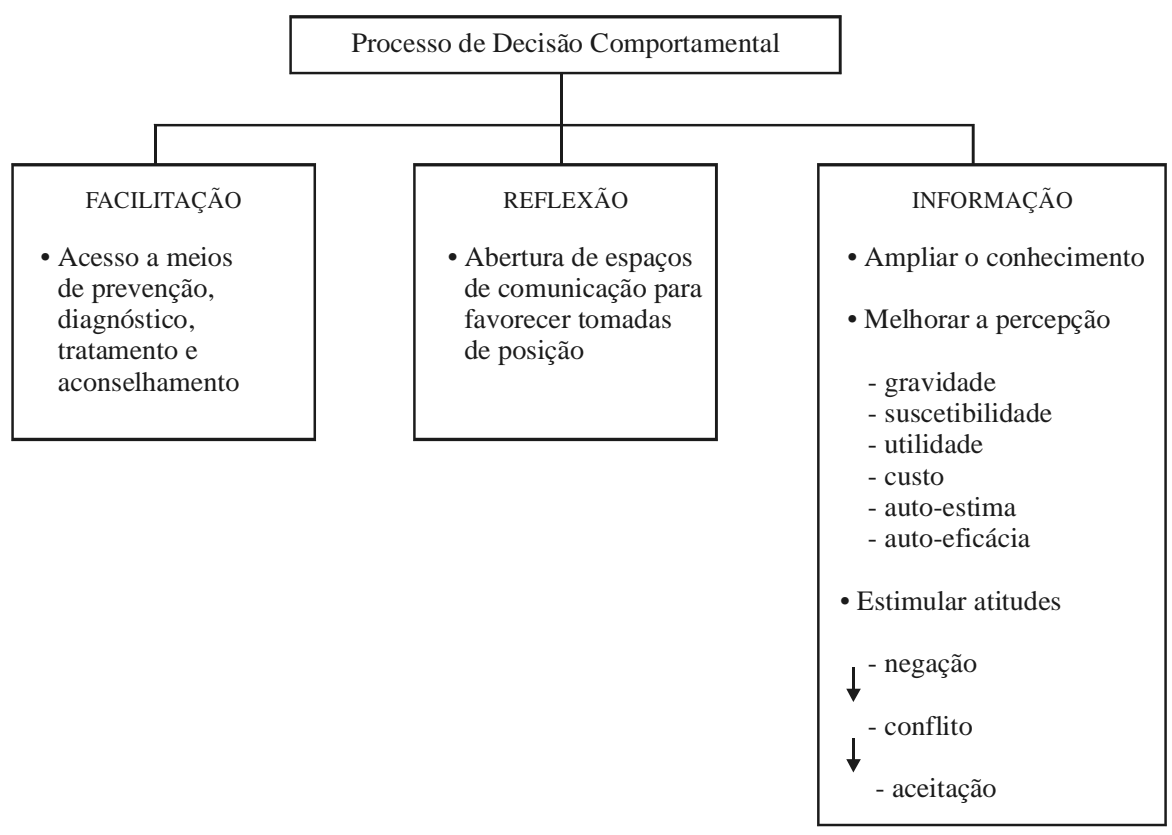

\section{INFORMAÇÃO E AIDS}

Considerando que, em relação aos três el ementos constitutivos do trabalho educativo que propusemos, os aspectos de facilitação e reflexão já foram suficientemente abordados ao longo do texto, gostaríamos de aprofundar um pouco mais o aspecto específico da informação a ser transmitida nesta prática. Para isto, aproveitaremos alguns elementos teóricos contidos no Health Belief Model (HBM) de Janz \&
Becker (Mehriar \& Carballo, 1990) e no PRECEDE Model (Green \& Krenter, 1991; Mehriar $\&$ Varballo, 1990). Desta forma, a veiculação de informações relativas ao HIV, nos seus diversos níveis, deve considerar três aspectos sobre os quais inevitavelmente exercerá alguma influência: os conhecimentos das pessoas e suas "falhas" (como a questão do mosquito), suas percepções e as atitudes desta frente à doença. Estes três aspectos são certamente interligados, mas, mesmo correndo o risco da 
segmentação, podemos tentar identificá-los mais especificamente.

Os conhecimentos sobre AIDS, como quaisquer outros, refletem o terreno efetivo e cultural em que a informação é lançada (e, portanto, o campo das percepções e atitudes). Desta forma, voltando à questão do mosquito, é possível supor que esta falha tenha relação com uma percepção fatalista da doença (já que ninguém pode, efetivamente, se prevenir com segurança das picadas) e também, ao menos no caso do Brasil, com a veiculação de informações sobre o dengue ou outras doenças provocadas por este vetor que poderiam favorecer a confusão (Fernandes et al., 1992). Diante disto, é preciso esclarecer a questão de forma direta e objetiva, mesmo considerando que isto não vá solucionar, por si só, a percepção fatalista supostamente presente. Possivelmente, esta postura contribuirá para o enfrentamento de resistências, tornando as pessoas mais acessíveis a outras mensagens. Algo como uma "limpeza de terreno". É importante, todavia, na avaliação das informações a serem transmitidas, o conhecimento prévio destes pontos "falhos" (que podem ser acessados através de inquéritos CAP, conversas, grupos focais etc), procurando-se esclarecê-los diretamente, sem deixar de considerar suas implicações nos outros campos.

A percepção que as pessoas têm sobre o HIV/ AIDS, influencia fortemente não só a própria quantidade e qualidade de informação que é efetivamente assimilada, como se reflete nas atitudes assumidas frente à questão. Trata-se, portanto, de um aspecto fundamental a ser considerado na avaliação da informação a ser fornecida. Em relação a isto, o HBM propõe quatro tipos básicos a serem avaliados: A percepção da severidade ou gravidade do problema; a percepção da suscetibilidade (possibilidade de adquirir a infecção); a percepção de utilidade (o sentido de benefício que a modificação de hábitos pode provocar), e a percepção de custo (isto é, as inconveniências e problemas que esta modificação de hábitos pode causar). Julgamos importante acrescentar a estes a noção de auto-estima (Peterson \& Szterenfeld, 1992) e auto-eficácia (McAlister et al., 1991), esta última relacionada à autoconfiança em conseguir efetuar a mudan- ça pretendida. Desta forma, as informações a serem divulgadas devem ser avaliadas em função de seu possível impacto nestes campos de percepção, em seu conjunto.

Como já mencionados, as atitudes das pessoas frente à AIDS se relacionam diretamente, embora não exclusivamente, com o conhecimento e a percepção que elas possuem do problema. O campo das atitudes revela, entretanto, de forma mais nítida, o aspecto histórico e processual da questão da mudança de comportamentos, daí sua importância na avaliação da informação a ser fornecida. Isto é, o conjunto de conhecimento e percepções sobre o HIV influencia a adoção de determinadas posturas dentro de um processo pessoal A (ou social) de relacionamento com a questão, e a informação veiculada poderá, portanto, ser mais ou menos adequada ao avanço deste processo. Considerando isto, temos observado três tipos de atitudes distintos, embora muitas vezes interligados no movimento de relação com a epidemia: o primeiro se caracteriza por um alheamento do problema, através da negação da auto-susceptibilidade ao HIV. O segundo é decorrente da visualização do problema, isto é, da percepção da susceptibilidade e severidade, e é muitas vezes deflagrado pelo contato com um doente, pela conversa regular com um colega ou pela divulgação de notícias ou informações epidemiológicas. Neste momento surge freqüentemente o pânico da doença, e emergem suas associações com a questão da morte e outras de conteúdo fantástico, ligadas possivelmente à sexualidade, punição etc. $\mathrm{O}$ terceiro tipo se caracteriza por uma desmistificação e aceitação da realidade, onde são traçadas as estratégias pessoais de prevenção.

Estas observações sobre as atitudes frente ao HIV condensam, de certa forma, os estágios de mudança referidas por O'Reilly \& Higgins (1991), que, utilizando conceitos oriundos de três fontes teóricas distintas (Health Belief Model, Theory of Reasoned Action e Social Learning Theory), propõem um modelo de estagiamento do processo de mudança comportamental relacionado ao HIV. Este modelo, já utilizado em programas para redução do tabagismo e de doenças cardiovasculares, consiste em seis estágios: pré-contemplativo (ausência de intenção de mudar o comporta- 
mento), contemplativo (intenção de mudança a longo prazo); ação iminente (intenção de mudança a curto prazo); ação (tentativa de mudança); manutenção (mudança de comportamento a longo prazo) e reincidência (que pode provocar o reinício do processo ou simplesmente estacioná-lo). Acreditamos, entretanto, que este estagiamento é de difícil observação e aplicação práticas, ao menos em nossa realidade, onde o acompanhamento e monitoramento dos trabalhos educativos raramente é feito com a infra-estrutura necessária. É preciso, por outro lado, avaliar com cuidado até que ponto se pode identificar uma similaridade nos processos de mudança relacionados a temas com características psicossociais tão distintas, como o tabagismo, a obesidade e a AIDS. Finalmente, consideramos que a mudança de atitude em relação à epidemia do HIV não se processa de forma linear, através de estágios, mas antes através de um dinâmico e instável processo de aprofundamento progressivo destes diversos movimentos no decorrer do tempo. Desta forma, a informação a ser veiculada nos trabalhos educativos individuais, grupais e coletivos deve ter em conta as atitudes que estão sendo tomadas, buscando-se compreendê-las enquanto um processo e fortalecer seu aprofundamento através da identificação de problemas relacionados à percepção existente sobre a questão e às lacunas verificadas no seu conhecimento.

Um último comentário, a título de ilustração, em relação ao conteúdo das informações relacionadas ao HIV, é a forma como são associadas, nas mensagens educativas, a sexualidade, a morte, o desconhecido, o incurável, o trágico, o doente, o feio. Consideramos que este coquetel deva ser tratado com o maior cuidado, já que a sensação de impotência que ele freqüentemente provoca não combina, de modo algum, com a positividade e vitalidade do sexo. Embora tenhamos a compreensão da realidade destes aspectos, julgamos importante buscar uma informação relativa e progressivamente mais normalizada sobre a AIDS, cujos meios de prevenção podem ser apresentados de um modo mais vitalizado e positivado. Afinal, esta não é a única doença incurável de nossa época, nem está relacionada, ao menos biologicamente, à impotência sexual.

\section{CONCLUSÃO}

$\mathrm{O}$ fato de vivermos cotidianamente influenciados pelo marketing e condicionados em maior ou menor grau a operarmos determinados comportamentos não é um argumento suficiente para acreditarmos ou aderirmos a um trabalho educativo centrado nestas mesmas estratégias. $O$ papel do subjetivo e peculiar de cada indivíduo ou grupo deve ser reconhecido, valorizado e explicado, mesmo que, em função de sua própria natureza, dificilmente possam ser controlados e avaliados cientificamente. "O cotidiano não é científico" (SES-RJ, 1992). O que cada um vai fazer em relação à AIDS será sempre uma questão pessoal e íntima, e este fato não deve ser atenuado por ilusões onipotentes comportamentalistas. Cabe-nos, como profissionais de saúde, fazer nossa parte, da melhor forma possível, e acreditar na capacidade das pessoas de desejar e buscar uma melhor condição de vida, apesar de todas as dificuldades que, aliás, também são as nossas, enquanto seres humanos.

\section{RESUMO}

FERNANDES, J. C. L. Práticas Educativas Para a Prevenção do HIV.AIDS: Aspectos Conceituais. Cad. Saúde Públ., Rio de Janeiro, 10 (2): 171-180, abr/jun, 1994.

A importância e complexidade do trabalho educativo voltado para prevenção do HIV demandam o constante aprofundamento de seus conteúdos. Do ponto de vista ideológico, é preciso levar em conta que, com a epidemia, a medicina alcança um terreno até então relativamente preservado do seu projeto hegemônico: a vida sexual das pessoas. Uma postura normatizadora coercitiva em relação a este campo pode levar a uma reação prejudicial à adoção de comportamentos mais seguros. Para evitar este tipo de prática, deve-se considerar a importância da participação e autoresponsabilização dos indivíduos na prevenção da infecção. O estado deve também assumir sua própria responsabilidade em garantir os meios de facilitação necessários à mudança de comportamentos. 
A partir destas premissas, o autor propõe três aspectos a serem avaliados no planejamento dos trabalhos educativos destinados à prevenção da AIDS: a informação a ser divulgada, e seus efeitos sobre os conhecimentos, percepções e atitudes da população frente ao HIV; a facilitação da resposta e a abertura de espaços para reflexão individual e coletiva.

Palavras-Chave: educação em Saúde; AIDS; HIV; Prevenção

\section{REFERÊNCIAS BIBLIOGRÁFICAS}

BOLTANSKI, L., 1991. As Classes Sociais e o Corpo. Rio de Janeiro: Graal.

CLAVREUL, J., 1983. A Ordem Médica. São Paulo: Brasiliense.

DNE (Dirección Nacional de Epidemiologia) \& MINSAP (Ministério de Salud Pública), 1992. Programa Naacional de Prevención y Control del SIDA.Cuba: Minsap.

FERNANDES, J. C. L.; COUTINHO, E. S. F. \& MATIDA, A. H., 1992. Conhecimentos e atitudes relativas à SIDA/AIDS em uma população de favela do Rio de Janeiro. Cadernos de Saúde Pública, 8: 176-182.

FREIRE, P., 1975. Pedagogia do Oprimido. Rio de Janeiro: Paz e Terra.

1978. Educação como Prática da Liberdade. Rio de Janeiro: Paz e Terra.

GREEN, L. W. \& KRENTER, M. W., 1991. Health Promotion Planing: an Educational and Enviromental Approach. $2^{\text {nd }}$ ed., Mountain View: Mayfield Pub. Co.

KELLY, J. A. \& LAWRENCE, J. S. St., 1990. The impact of community-based groups to help persons reduce HIV infection risk behaviours. AIDS Care, 2: 25-36.

KELLY, J. A.; LAWRENCE, J. S. St.; HOOD, H. V. \& BRASFIELD, T. L., 1989. Behavioral intervention to reduce AIDS risk activities. Fournal of Consulting and Clinical Psychology, 57: 60-67. Medicine et Hygiène, 1991. Encore bien loin de la victoire sur le SIDA. Médecine et Hygiène, 49: 3346 (Editorial).

McALISTER, A. L., 1991. Population behaviour change: a theory-based approach. Fournal of Public Health Policy, autumm, pp. 345-361.
McALISTER, A. L.; PUSKA, P.; ORLANDI, M.; BYE, L. L. \& ZBYLOT, P. L., 1991. Behaviour Modification: Principles and Illustration. $2^{\text {nd }}$ ed., vol. 3, Oxford: Oxford University Press.

MEHRIAR,A.H.\&CARBALLO,M., 1990. Models of Behaviour Change: Implications for Research and Interventions Programmes for Prevention and Control of HIV and AIDS. World Health Organization, Global Programme on AIDS, Geneva: WHO.(Mimeo.)

MS (Ministério da Saúde), 1992. Boletim Epidemiológico sobre AIDS. Ano V, $\mathrm{n}^{\circ}$ 6, Brasília: Programa Nacional de Doenças Sexualmente Transmissíveis/AIDS, Ministério da Saúde.

MUNHOZ, R.; GRANJEIRO, A. \& SILVA, J. L.; 1989. Pesquisa "Você e a AIDS". Programa de Controle e Prevenção contra a AIDS, Setor de Vigilância Epidemiológica do Centro de Referência e Treinamento AIDS-SP, São Paulo. (Mimeo.)

O’REILLY, K. R., 1991. What we Have Learned from AIDS Community Demonstration Projects. Center for Diseases Control, Public Health Service. (Mimeo.)

O'REILLY, K. R. \& HIGGINS, D. L., 1991. AIDS community demonstration projects for HIV prevention among hard-to-reach groups. Public Health Reports, 106: 714-720.

PARKER, R., 1987. Acquired immunodeficiency syndrome in urban Brazil. Medical Anthropological Quartely, 1: 155-175.

, 1991. Depois da AIDS: mudanças no comportamento (homo)sexual. In: AIDS a Terceira Epidemia (H. Daniel \& R. Parker), pp. 102-114, São Paulo: Iglu.

PETERSON, C. \& SZTERENFELD, C., 1992. Organizing a project with community-based health agents recruited from prostitutes in Rio de Janeiro. Public Health, 106: 217-223.

PS.AMABB (Posto de Saúde da Associação dos Moradores e Amigos do Bairro Barcelos), 1991. Relatório do Programa Comunitário de Controle da AIDS. Posto de Saúde da AMABB. Rio de Janeiro: OS.AMABB. (Mimeo.)

RIFKIN, S. B. \& WALT, G., 1986. Why health improves: Defining the issues concerning 'comprehensive primary health care' and 'selective primary health care'. Social Science and Medicine, 23: 559-566. 
SANTANA, S.; FAAS, L. \& WALD, K., 1992.

Human immunodeficiency virus in Cuba: The public health response of a third world country. In: A Porfolio of AIDS/STD Behavioral Interventions and Research (L. S. Bond, ed.), pp. 87-93, Washington, D.C.: PAHO.
SES-RJ (Secretaria de Estado de Saúde do Rio de Janeiro), 1992. Diretrizes Gerais das Práticas Educativas na Prevenção do HIV/ AIDS. Documento Preliminar, Secretaria de Estado de Saúde do Rio de Janeiro, Divisão de Educação em Saúde. Rio de Janeiro: SES/RJ. (Mimeo.). 